Original Research Paper

\title{
Processability of Bulk Metallic Glasses
}

\author{
${ }^{1}$ Raffaella Aversa, ${ }^{1}$ Daniela Parcesepe, ${ }^{2}$ Relly Victoria V. Petrescu, ${ }^{3}$ Guanying Chen, \\ ${ }^{2}$ Florian Ion T. Petrescu, ${ }^{1}$ Francesco Tamburrino and ${ }^{1}$ Antonio Apicella \\ ${ }^{1}$ Advanced Material Lab, Department of Architecture and Industrial Design, \\ Second University of Naples, 81031 Aversa (CE) Italy \\ ${ }^{2}$ ARoTMM-IFToMM, Bucharest Polytechnic University, Bucharest, (CE) Romania \\ ${ }^{3}$ Harbin Institute of Technology and SUNY Buffalo, China
}

Article history

Received: 03-01-2017

Revised: 04-02-2017

Accepted: 14-02-2017

Corresponding Author: Florian Ion T. Petrescu ARoTMM-IFToMM, Bucharest Polytechnic University, Bucharest, (CE) Romania Email: scipub02@gmail.com

\begin{abstract}
Microfluidic Shear Flow Instabilities in Injection Molded Glassy Metal are investigated. The formation of microfluidic shear flows instabilities involving the presence of different viscosities fluids has been observed in injection molded Bulk Metallic Glasses. The complex rheology of injection molded metastable glassy metal, which has been hypothesized to induce selective clustering of atoms of different steric hindrance, is discussed. Smaller $\mathrm{Be}, \mathrm{Cu}$ and $\mathrm{Ni}$ atomsmay differently rearrange themselves in the bulk metal glassy super cooled liquids forming flow streams of lower viscosity. Segregation of atoms of different size could activate a variety of viscous flow instabilities such as folding and swirling. FEI Scios Dual-Beam Electron scanning and optical microscopy observations of a commercial liquid metal alloy $\left(\mathrm{Zr}_{44} \mathrm{Ti}_{11} \mathrm{Cu}_{10} \mathrm{Ni}_{10} \mathrm{Be}_{25}\right)$ have been carried out. We discussed the influence of short-range order clusters distribution and its effect in locally induced shear flow instability and corrosion resistance.
\end{abstract}

Keywords: Bulk Glass Metals, Rheology, Shear Flow, Segregation, Microfluidic Instability

\section{Introduction}

Bulk Metallic Glasses (BMGs), even called bulk amorphous alloys, are a category of new advanced materials with a disordered atomic-scale structure. Their unique microstructure imparts outstanding physical and chemical properties (Huang et al., 2016) to the manufacts (Schroers, 2010b; Schroers et al., 1999).

The absence of long-range atomic order lead to unique, physical, chemical and mechanical properties of bulk metallic glasses, that have found pilot applications in a wide range of field from sporting goods to precision mechanical components, electronics and biomedicine (Wang et al., 2004; Schroers, 2010a; Kumar et al., 2009; Inoue and Takeuchi, 2011).

Zr-based BMGs are technologically attracting since they can be processed in larger parts due to their broad super-cooled liquid region and high glass-forming ability. Among the Zr-based alloy systems recently developed, containing different combination of $\mathrm{Cu}, \mathrm{Al}$, Ti, Ni (Wang, 2007; Shen et al., 2005; Jiang et al., 2008; Liu et al., 2007; Mattern et al., 2002), we investigate a commercial BMG containing the same atoms but with the addition of Be. In the last few years Zr-based bulk materials have been deeply explored for their superior Glass Forming Ability (GFA). The properties advantage ranges from high mechanical strength, high fracture strength, superior elastic limit to good and precise deformability, good ductility, low coefficient of thermal expansion and excellent corrosion/wear resistance (Aversa et al., 2016a; 2016b; 2016c; 2016d; 2016e; 2016f; 2016g; 2016h; 2016i; 2016j; 2016k; 2016l).

BMGs multicomponent alloys based on $\mathrm{Zr}$ have superior GFA and they can be produced into parts with thickness larger than a few centimeters by conventional melting and casting techniques (Liu et al., 2002).

Among the other advantage, BMGs utilization offers reduced process costs and it gives the possibility to manufacture a variety of industrial products (Morito and Egami, 1984).

The microstructure of BMGs compared to the other conventional metals holds the advantage of not presenting a long-range order crystalline structure. Conversely, their microstructure has a short-range organized amorphous arrangement, which is 
characteristic of all glassy materials, such as those found in ceramics and polymers (Busch, 2000).

An important issue in the processing of glass forming materials is, therefore, the rheology of the melt during the cooling procedures.

This issue is particularly critical for thermodynamically favored crystal forming materials. For these materials, such are metals, sufficiently high cooling rates are necessary to avoid crystallization from the melt and to preserve the liquid amorphous microstructure (Huang et al., 2016) in the solidified glass (Debenedetti and Stillinger, 2001; Eckert et al., 1998).

Therheology of the melt, which describes the basic intermolecular and interatomic interactions occurring in the liquid phase, has been deeply investigated and different phenomenological as well theoretical models have been presented in literature (Eyring, 1936; Glasstone et al., 1941).

A number of different models to predict the viscosity of liquids have been developed by Eyring (1936). One of the modifications considering atomic structure, which better adapts to metallic melts, was derived as:

$\Delta G^{*}=\frac{16}{3} \pi \gamma^{3}\left(\frac{V_{m}^{c}}{\Delta G_{m}+E_{c}+P \Delta V_{m}}\right)$

where, $d_{i}$ is the diameter of the space occupied by the atomic species. These approach has been validated for single metal or simple bi-metallic alloys. Three or more metal atoms of different size and physical properties usually form glassy metal structures and, therefore, a more complex modeling should be applied.

This atomic/molecular dynamics approach, which is in contrast to hydrodynamics, does not utilize constitutive state equations or direct continuum-level equations, but it is directly based on interatomic force laws (Cubaud and Mason, 2012) from which the effects of the inertial to viscous forces governing the melt flow are derived.

Flow instabilities can be generated at different size scale in fluids in motions (Fig. 1). Traditional hydrodynamics can describe the Kelvin-Helmholtz (Fig. 1d) and Kármánvortex street like patterns (Fig. 1d).

Molten BMG hydrodynamics evolution of microfluidic turbulence in complex composition alloy can be quantitatively evaluated, instead, in terms of molecular dynamics of alloy atoms interactions and deriving flow characteristics.

Future evolution of computing resource power involves that any increase in the simulation computational complexity should not be necessarily accompanied by a decrease in the size of the system to be modelled.

A computational tool where size scale is no longer an issue will lead to a potential increase of the handling complexity that would greatly enhances the fidelity of simulations.

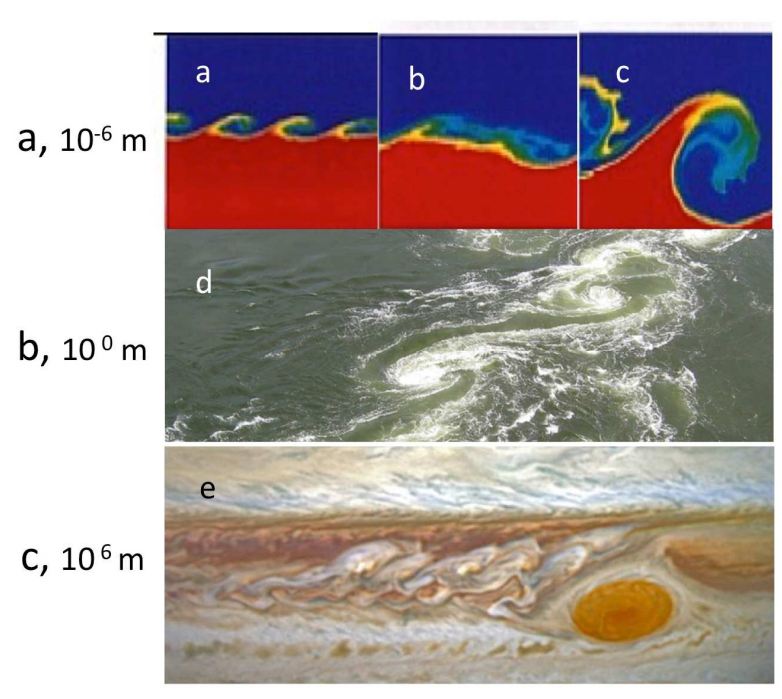

Fig. 1. Instability Flow patterns at different size scale: (a, b, c) Microfluidic flow patterns evolution in two flowing adjacent fluids (Alchorn, 2008); (d) water flow instabilities at scal of a meter; (e) Gas flow instabilities on Jupiter atmosphere at hundred thousand meter scale

Future machine could enable the full tissue engineering modelling of a human cell or group of cells overcoming the technological barriers, as it has already been occurring for the pioneering works by our research group (Annunziata et al., 2006; Apicella et al., 2010; 2015; Apicella and Aversa, 2016; Aversa et al., 2009; 2016b; 2016c; 2016d; 2016e; 2016f; 2016g; 2016h; 2016i; 2016j; 2016k; 2016l; Sorrentino et al., 2007), where we evaluated the connections at atomic, molecular and cellular scales of physics, chemistry and biology.

High-performance molecular dynamics codes have been proposed (Alchorn, 2008) to investigate the formation and evolution of the Kelvin-Helmholtz microfluidic flow instability using molecular dynamics techniques (Fig. 1a-c).

It has been reported by (Gostin et al., 2010; Aversa et al., 2016e) that the difference in the atomic radius and the free energy of mixing among the components favour the generation of inhomogeneous rather than uniform structures with chemical and morphological short and medium-range orders leading to localized compositional segregation or phase separation (Aversa et al., 2016e).

In addition to these chemical-physical properties, the processing implications on local rheology and the deriving microfluidic flow instabilities could further favor phase segregation and are investigated and discussed in this study (Trachenko, 2008).

In the specific, we analyze here the result of morphological microscopic observation made by optical and Ion and electron microscopy on injection molded glassy metal plate. 


\section{Materials and Procedures}

A commercial Bulk Metallic Glass of atomic composition $\mathrm{Zr}_{44} \mathrm{Ti}_{11} \mathrm{Cu}_{10} \mathrm{Ni}_{10} \mathrm{Be}_{25}$ (LM001B, Liquid Metals Technologies Inc, Ca USA), in the form of a 3 and $2 \mathrm{~mm}$ thick injection molded plate (Engel injection molding machine operating at $1050-1100^{\circ} \mathrm{C}$ ) of dimension of $13 \mathrm{~mm}$ per side has been used (Fig. 2, left hand side).

The samples for microscopy observations were cut from the plate by water jet. Three areas of the sample were investigated. The transverse and surface of cut samples (figure and a third surface area where an evident molding defect was visible. FEI Scios Dual-Beam Ion and electron Microscope and a Leitz optical microscope have been used. Surface etching has been carried out on the sample using Methanol (33.3\%), Hydrochloric acid (33.3\%), Nitric acid (33.3\%), Hydrofluoric acid $0.1 \%$.

In particular, we examine for surface defects (Fig. 2, right hand bottom) and presence of microfluidic flow instability patterns. Internal surface morphology and crosssections of the surface defect were made by means of FEI Scios Dual Beam using a Focused Ion Beam (FIB) for cross sectioning and Scanning Electron Microscopy (SEM) for morphological analysis and chemical composition analysis (EDS, Energy Dispersive Spectrometry).

\section{Results and Discussion}

The Scanning Electron Microscopy of the surface revealed the presence of surface groove shaped defects that are characteristics of flow instabilities observed in polymeric injection molded parts (Fig. 3). These melt flow instabilities are generally associated to the advancing front filling the mold.

Kelvin-Helmholtz (KH) flow instability is described to appear when adjacent fluid layers undergo high shear forces and it testifies the transition from smooth to turbulent flow similar to those that can be seen in the Great Red Spot and other fluid vortices in Jupiter's atmosphere (Fig. 4a).

However, in our case the flow instability occurs at micro-scale level (Fig. 5 left hand side).

A Focused Ion Beam (FIB) cross-section of the groove defect is shown in Fig. 5. The shape of the crosssectioned groove shows the ripening and overlapping (Fig. 5 right) of the metal molten flow.

Similar surface defects are observed in polymer injection molding parts when the mold is too cold and/or the advancing front slow down leading to early polymer vitrification or excessive high viscosity build-up. Surface defect in glassy metal injection molded parts, may be due to the same reason. An excessively cold mold, in fact, could generate high temperature gradients through the molten metal (Fig. 9) leading to high viscosity changes that increases the possibility of microfluidic flow instability and micro-groove and ripples formation (indicatively reported in Fig. 9) such as that seen in Fig. 3 (right top) and 5 .

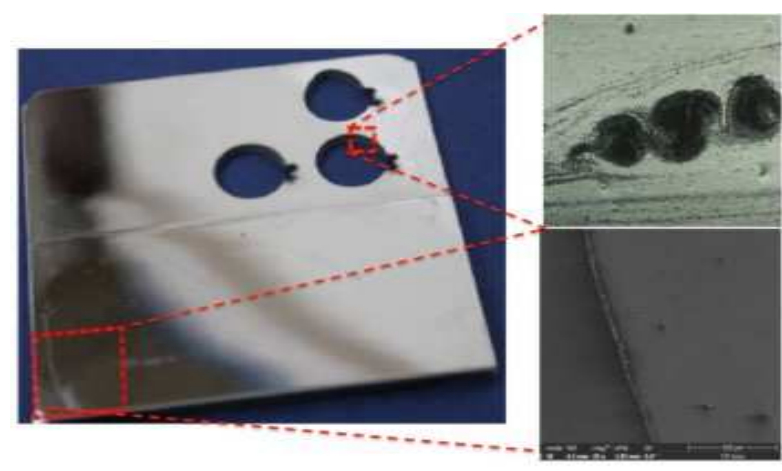

Fig. 2. Injection molded BMG sample plate (left): Microfluidic pattern in the sample section (right up) and a surface micro-defect (right bottom)

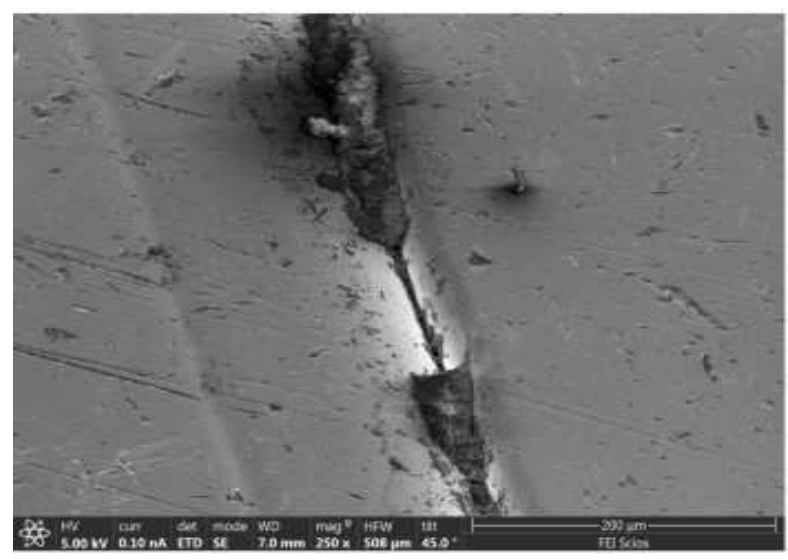

Fig. 3.Surface micro defect on the Injection molded BMG sample plate (left)

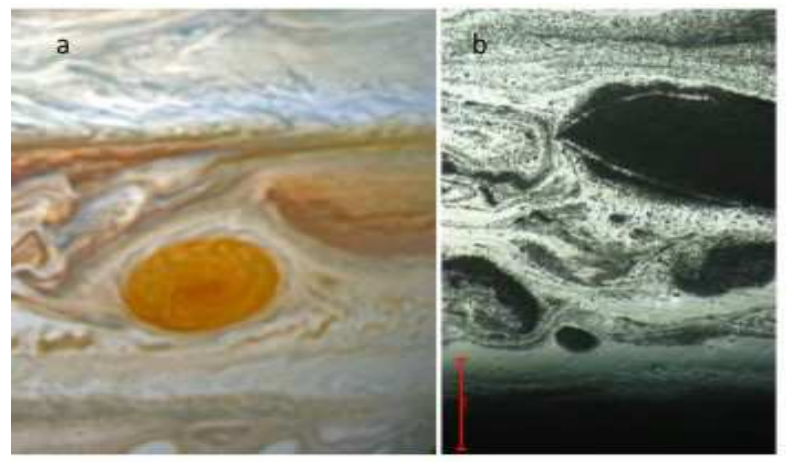

Fig. 4. Kelvin-Helmholtz flow instability (a) Jupiter Great Red Spot; (b) surface optical microscopy after acid attack of the sectioned injection molded BMG slab (scale 500 microns)

The cooling rate, in fact, is lower in the center than in the external areas of the sample and this condition increases the formation of a short-range order and therefore of crystalline-phase grains. Moreover, in injection made parts, high shear stresses may be induced by the rheology of the molten alloy in the mold surface proximity where a region, neighboring the solidified glass, a still fluid but highly viscous layer exists (Fig. 9). 


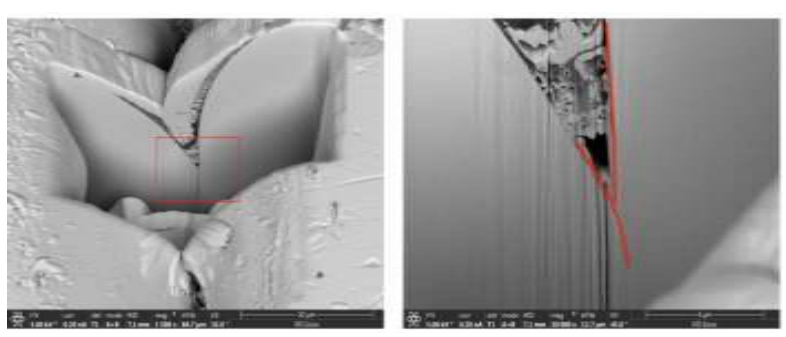

Fig. 5. Focused Ion Beam cross-section of the groove defect (left side) and flow fronts overlapping (right side, red lines)

The groove surface defects, then, can be attributed to molten BMG microfluidic flow in the mold that has been frozen by the vitrification process occurring at the interface with the cold mold surface. However, some doubts still remain about the composition inside the flow instability lines.

Figure 2 (Top right), Fig. $4 \mathrm{~b}$ and $6 \mathrm{~b}$ show optical microscopy images of the acid etched surface of the section of the injection-molded sample.

The morphological development of the interface between two different velocities flows experiences transient regimes (Fig. 2a-c), in which the interface of the dominant structure grows in size with the same diffusion controlled kinetic (Fig. 2c). The theoretical shape of the dominant $\mathrm{KH}$ structure evaluated using the molecular dynamics approach is compared in Fig. 6 with the flow patterns made evident by acid attack of the plate section surfaces (Fig. 6 right). Generalizations of the momentum thickness formula have been suggested by (Alchorn, 2008) where the interface grows at the square root of time in very early stages (hundred of ps) with a regime associated with momentum diffusion.

The variety of fluctuations initiated at the atomistic level leads to momentum diffusion and vortex regimes (Fig. 2a) that are characteristic of continuum hydrodynamic behaviour even at much higher size scale (Fig. 4a and 4b). As it occurs in Jupiter clouds patterns at $10^{6}$ meter scale (Fig. $2 \mathrm{a}$ and $4 \mathrm{a}$ ), or in river water flow at a 1 meter scale (Fig. 2d). When fluids with different densities and viscosities move relative to one another they lead to shear flow instabilities along the interface between these two fluid layers. In the same manner, atomistic fluctuations induce similar instability patterns on a $10^{-3}$ meter scale (Fig. 5a-c). It can be recognized in Fig. 7 (right) a classical Kármán vortex streetpattern (Fig. 7 left), which is formed by repeating swirling vortices produced by the unstable separation of flow of a fluid around an obstacle.

Vortex street formation occurs at a specific narrow ratio between inertial to viscous forces, which is numerically represented by the dimensionless Reynolds numbers (Re) and reaches typically values of about 90 . The Reynolds number relates the free stream speed of the undisturbed flow and the local fluid flow speed in the vicinity of a disturb, namely:

$$
R_{e}=\frac{U L}{v_{0}}
$$

where, $U$ is the speed of the undisturbed free stream (Fig. 9), L is the characteristic length of the disturb (or interacting solid as it could be a mould internal space thickness reported in Fig. 9) and is the kinematic viscosity $\mu_{0}$ (the ratio between the fluid density and its dynamic viscosity).

For common fluids in isothermal conditions, $\mathrm{Re}$ number essentially depends on the fluid dynamic viscosity, while, for multicomponent fluids, such are the BMG molten alloys, different densities fluids can be formed it depends also on the fluid density. In these liquids, in fact, thermodynamically favored local segregation may occur in compressed and high sheared melt flows (Apicella and Aversa, 2016; Aversa et al., 2016f) leading to the formation of fluids of different densities. Segregation of atoms of different steric hindrance and electro-negativities will then induces in the flowing meltvariations of both the inertial forces (which depend on local densities) and viscosity forces (which depend on local interatomic interactions in the molten alloy). Local fluid instabilities are then generated.

Flow instabilities, in the form of Kármánvortex street like patterns, are present in the optical micrographs of sample surfaces reported in Fig. 4b, 6b, 7a and 8. The instability may be described as Kelvin-Helmholtz shear flow that are generated by the dissimilar rheology behavior of two fluid layers with different densities and viscosities on top of each other. Layers of different densities and viscosities moving at different speeds produce what is called "velocity shear" across the interface between the two fluids.

Aversa et al. (2016a) described metal atom segregation in the BMG alloy when subjected to highly sheared adjacent flows (Fig. 9). High shears reduce the free energy of mixing thermodynamically favoring the creation of more ordered structures with compositional difference within the bulk alloy. The resulting density and viscosity differences can then induce local KelvinHelmholtz instabilities such as those observed in the optical microscopy of Fig. 4, 6 and 7.

According to this atomic level approach, which is in contrast to hydrodynamics, molecular dynamics does not utilize constitutive state equations or direct continuum-level equations but it is directly based on interatomic force laws. The new approach proposed by Alchorn (2008) to microfluidic flow dynamics only considers the physical nature of inter-diffusion at interfaces. Flow dynamic relationships are not more considered as numerical artefacts relating density with temperature, pressure and others transport properties (diffusion, viscosity and surface tensions), but they are directly derived from the basic interatomic forces. 

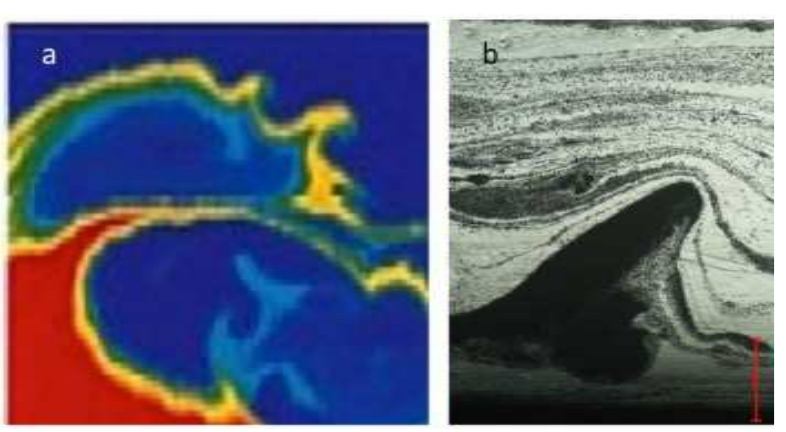

Fig. 6. Flow instability evolution (a) Water jet debris removal (b) on the injection molded BMG slab analyzed using Dual Beam Microscopy
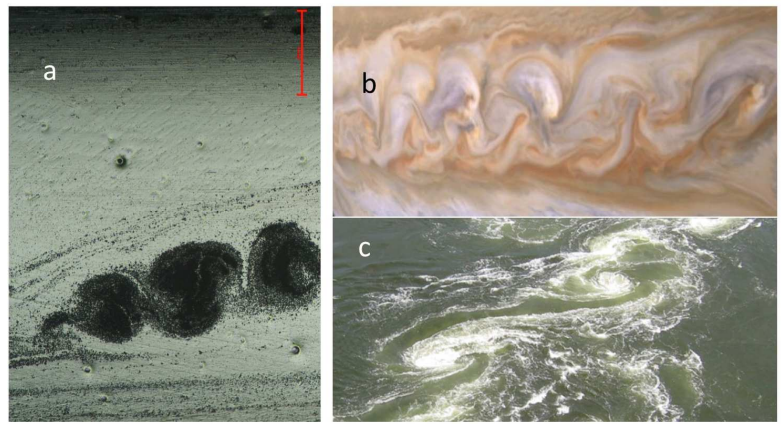

Fig. 7. Corrosion micro path in etched BMG injection molded plate (a); characteristic flow instabilities in Jupiter atmosphere (b) and river water (c)
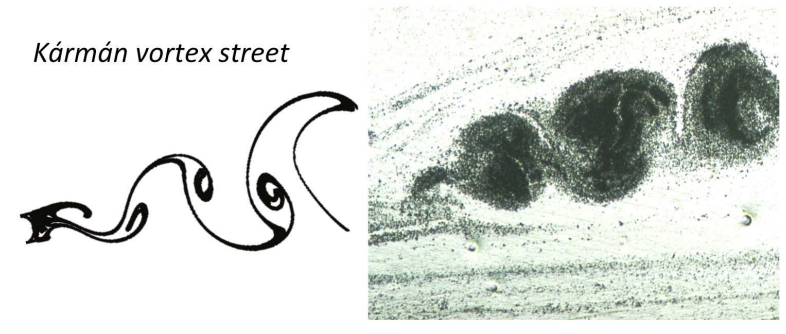

Fig. 8. Kàrmànvortez street (Left) and optical microscopic view of the corrosion path (Right)

Instead adding additional degrees of freedom to obtain converged results in hydrodynamic simulations, Molecular Dynamics approach overcomes the constraints imposed by using high computational resources (Alchorn, 2008). Surface etching of sample surface and section has made evident the presence of preferential corrosion path (dark areas in the Fig. 2, 4, 6 and 8). Flow instabilities patterns can be recognized in the corrosion pitting observed. In particular, it can be hypothesized that flow instabilities favor the further segregation of $\mathrm{Cu}$ rich phases that are more prone to etching and corrosion.

Figure 8 compares the classical Kármán vortex street and the pattern found in an etched and differently corroded region of the plate section. It can be inferred from the similarity of the geometrical paths that corrosion differently interests the sample surface.

The EDS chemical composition analysis in different regions of the sample surface has shown that difference in compositions can be observed. Compositional segregation or phase separation in the BMG sample is confirmed by the localized corrosion by pitting corrosion instead of generalized one after acid attack.

Microfluidic simulation of flow instabilities at the interface between miscible fluids of different viscosity has suggested that atomic level inter diffusion initially leads to the broadening of the two fluids interface activating the development of wave structures (Fig. 1a) that vertically grow in amplitude (Fig. 1b) and crest to form micron-scale vortices in the direction of the flow (Fig. 1c and left side of Fig. 6).

Once the BMG is immersed in the acid solution, galvanic couples can form among these different composition regions resulting in the classical pitting corrosion patterns shown in the Electron microscopies reported in Fig. 10.

The EDS analysis has been limited to the $\mathrm{Zr}, \mathrm{Ti}, \mathrm{Cu}$ and $\mathrm{Ni}$ atoms.

Amorphous glass metal $\mathrm{Zr}$ atoms richer (51,3\%) regions were found at the external surface while it is reduced at 20-60 microns deep in the internal layers $(43,8 \%)$ where, conversely, the $\mathrm{Ni}$ and $\mathrm{Cu}$ atoms increased their concentration from about 15 to $18 \%$.

Moreover, zones of significantly different composition have also been observed. In particular, crystallites particularly rich in $\mathrm{Cu}$ and $\mathrm{Ni}$ (25\% each instead of $15 \%)$ and poor in $\mathrm{Zr}(37 \%)$ and others richer in $\mathrm{Zr}$ (47\%) with $\mathrm{Cu}$ and $\mathrm{Ni}$ equally at $18 \%$ have been found.

Generally, the $\mathrm{Zr}$ element can promote strong passivation of the $\mathrm{BMG}$, forming $\mathrm{Zr}$-oxide, which is highly protective against corrosion (Cai et al., 2012). Zr rich regions, then, could show a higher corrosion resistance than $\mathrm{Cu}$ rich phases that are more prone to corrosion in presence of chloride ions (Tam et al., 2007). The compositional differences observed in our samples could be attributed to the thermal and rheological behavior of the melt during the processing by injection molding.

The viscosity of BMG changes of many orders of magnitude upon cooling from the equilibrium liquid into metastable under cooled liquid preceding its glass formation. In particular, the high temperature melt viscosities can range from about $10^{-3} \mathrm{~Pa} \mathrm{~s}$ for monoatomic or simple binary liquids to $10^{2} \mathrm{~Pa}$ s for dense, multi-component systems like our glass forming alloy (Iida and Guthrie, 1998; Way et al., 2007).

These shear stresses, as it occurs for local crystallization in shear bands formed in highly deformed BMG's (Kanugo et al., 2004), considerably reduce the energy barriers for nucleation favoring atom clustering and incipient formation of nano and micro-crystalline phases. 


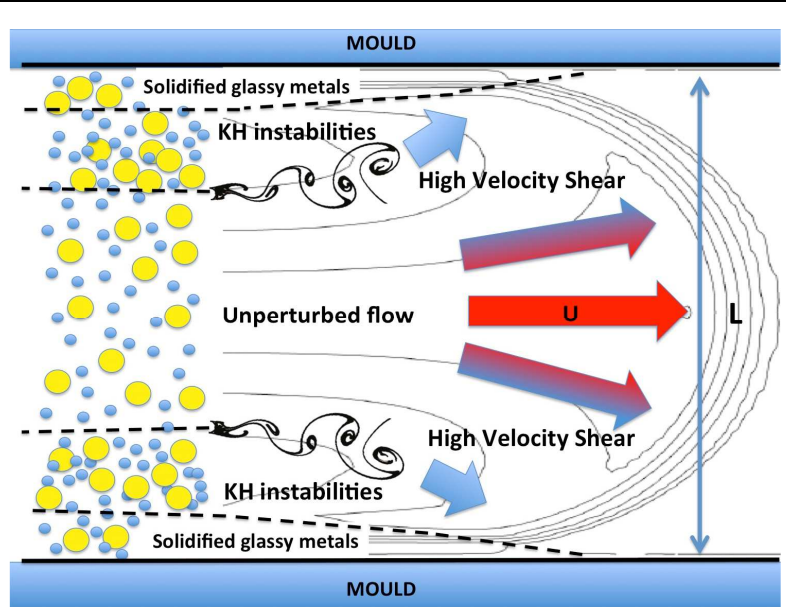

Fig. 9. High Velocity Shear regions build up in fountain flow front for injection molding of a $\mathrm{BMG}$

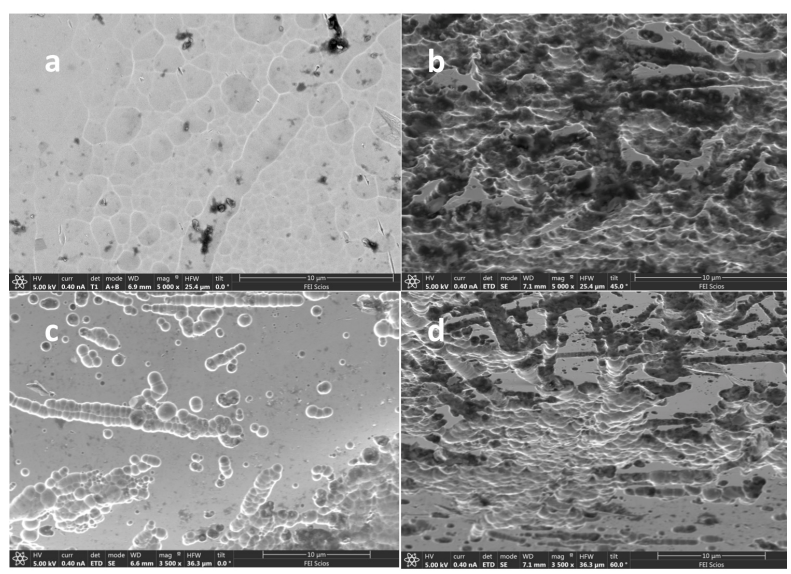

Fig. 10. Electron microscopy of etched BMG surfaces: Evidence of $\mathrm{Cu}$ rich phases by pitting by acid attack

In our samples sub-micron size crystal grains are observed in the layer between 20 and 40 microns from the external surface (Aversa et al., 2016b). In this interfacial layer (Fig. 8) two driving forces are acting to induce clustering, namely the high distance from the thermodynamic melting temperature and the higher proximity of the atoms that are squeezed and compacted by the intense high velocity shear stresses (reported in Fig. 9). According to a thermodynamic approach, proposed by Lee et al. (2006), the energy barrier $\left(\Delta G^{*}\right)$ for homogeneous nucleation from amorphous liquid is:

$\Delta G^{*}=\frac{16}{3} \pi \gamma^{3}\left(\frac{V_{m}^{c}}{\Delta G_{m}+E_{c}+P \Delta V_{m}}\right)$

With $\Delta G_{m}=$ molar free energy change in the transformation amorphous/crystalline phases, $T$ is the temperature, $P$ is the hydrostatic pressure, $\gamma$ is the interfacial free energy to form the critical size crystal nucleus) and $\Delta V_{m}$ is the molar volume change in the transformation between the amorphous $\left(V_{m}^{a}\right)$ and crystalline $\left(V_{m}^{c}\right)$ states. $E_{c}=\frac{1}{2} E \varepsilon^{2} V_{m}^{c} E_{c}$ represents the elastic strain energy induced by the volume change with E the elastic modulus and $\varepsilon=\left(V_{m}^{a}-V_{m}^{c}\right) / 3 V_{m}^{c}$. According to Equation 3, the presence of high velocity shear stresses increases in the region reported in Fig. 9, the compaction of the atoms producing molar volume decreases, reduction of the barrier energy $\Delta G^{*}$ with clustering and phase segregation that increases the possibility of generating flow instabilities.

\section{Conclusion}

Microfluidic shear flows instabilities, involving the presence of fluids of different viscosities, have been observed in our injection molded Bulk Metallic Glasses.

Our $\mathrm{Zr}_{44}-\mathrm{Ti}_{11}-\mathrm{Cu}_{10}-\mathrm{Ni}_{10}-\mathrm{Be}_{25} \mathrm{BMG}$, which is formed by different steric hindrance metal atoms, experiences significant temperature gradient and shear flow stresses when injected into a mold. This stress state thermodynamically favors the aggregation of similar size atoms inducing segregation of micro-fluidic phases of different densities and viscosities. A wide range of viscous flow instabilities, such as folding and swirling, can be then generated. It has been discussed how different steric hindrance atoms segregation occurs in BMG during the injection into a mold, melts having different viscosities are generated and flow streams can be formed. These streams rearrange themselves by forming complex stratified flow patterns that depend on the mould geometry and processing conditions that can be described by dimensionless Reynold number specific values intervals. Finally, as proven by microscopic observation, manufacturing process parameters, geometry, size and thickness could have a significant effect on the formation of microfluidic defects. The morphology, type and distribution of these defects will strongly depend on processing conditions. In fact, for more complex part and for manufacturing processes with a lower control of rheological parameters, changes in cooling rate within the part may cause build-up of excessive thermal gradients and severe flow instabilities generation, especially in the interlayer's between the solidified external glass metal and the still fluid, but near to its glass transition, highly shear strained melt.

\section{Acknowledgement}

The Authors acknowledge Liquid Metals Technologies Inc, Ca USAthat kindly supply the samples for the characterization and Dr Francesco Tatti (FEI Company Application Specialist SEM-SDB) for its contribut in the preparation of SEM analyses. 


\section{Author's Contributions}

All the Authors equally contributed to the experimental part and to the paper preparation.

\section{Ethics}

This article is original. Author declares that are not ethical issues that may arise after the publication of this manuscript.

\section{References}

Alchorn, A.L., 2008. In HPC Simulations, How Much is ENOUGH? SciDAC Review.

Annunziata, M., R. Aversa, A. Apicella, A. Annunziata and D. Apicella et al., 2006. In vitro biological response to a light-cured composite when used for cementation of composite inlays. Dental Mater., 22: 1081-1085. DOI: 10.1016/j.dental.2005.08.009

Apicella, D., R. Aversa, F. Ferro, D. Ianniello and A. Apicella, 2010. The importance of cortical bone orthotropicity, maximum stiffness direction and thickness on the reliability of mandible numerical models. J. Biomed. Mater. Res. Part B Applied Biomater., 93: 150-163. DOI: 10.1002/jbm.b.31569

Apicella, D., R. Aversa, M. Tatullo, M. Simeone and S. Sayed et al., 2015. Direct restoration modalities of fractured central maxillary incisors: A multilevels validated finite elements analysis with in vivo strain measurements. Dental Mater., 31: e289-e305. DOI: $10.1016 /$ j.dental.2015.09.016

Apicella and Aversa, 2016. Factors affecting chemophysical and rheological behaviour of $\mathrm{Zr}_{44}-\mathrm{Ti}_{11^{-}}$ $\mathrm{Cu}_{10}-\mathrm{Ni}_{10}-\mathrm{Be}_{25}$ metal glassy alloy supercooled. Am. J. Eng. Applied Sci., 9: 98-106.

Aversa, R., D. Apicella, L. Perillo, R. Sorrentino and F. Zarone et al., 2009. Non-linear elastic threedimensional finite element analysis on the effect of endocrown material rigidity on alveolar bone remodeling process. Dental Mater., 25: 678-690. DOI: $10.1016 /$ j.dental.2008.10.015

Aversa, R., F.I.T. Petrescu, R.V.V. Petrescu and A. Apicella, 2016a. Biofidel FEA modeling of customized hybrid biological hip joint design part II: Flexible stem trabecular prostheses. Am. J. Biochem. Biotechnol., 12: 277-285. DOI: 10.3844/ofsp.10936

Aversa, R., F.I.T. Petrescu, R.V.V. Petrescu and A. Apicella, 2016b. Biofidel FEA modeling of customized hybrid biological hip joint prostheses, Part I: Biomechanical behavior of implanted femur. Am. J. Biochem. Biotechnol., 12: 270-276.

DOI: 10.3844/ajbbsp.2016.270.276

Aversa, R., R.V.V. Petrescu, A. Apicella and F.I.T. Petrescu, 2016c. The basic elements of life's. Am. J. Eng. Applied Sci., 9: 1189-1197.

DOI: 10.3844/ajeassp.2016.1189.1197
Aversa, R., R.V.V. Petrescu, A. Apicella and F.I.T. Petrescu, 2016d. About homeopathy or "similia similibus curentur". Am. J. Eng. Applied Sci., 9: 1164-1172. DOI: 10.3844/ajeassp.2016.1164.1172

Aversa, R., R.V. Petrescu, A. Apicella and F.I.T. Petrescu, 2016e. One can slow down the aging through antioxidants. Am. J. Eng. Applied Sci., 9: 1116-1126. DOI: 10.3844/ofsp.10911

Aversa, R., E. Buzea, R.V.V. Petrescu, A. Apicella and M. Neacsa et al., 2016f. Present a mechatronic system having able to determine the concentration of carotenoids. Am. J. Eng. Applied Sci., 9: 1106-1111. DOI: 10.3844/ajeassp.2016.1106.1111

Aversa, R., R.V. Petrescu, R. Sorrentino, F.I.T. Petrescu and A. Apicella, 2016g. Hybrid ceramo-polymeric nanocomposite for biomimetic scaffolds design and preparation. Am. J. Eng. Applied Sci., 9: 1096-1105. DOI: 10.3844/ajeassp.2016.1096.1105

Aversa, R., V. Perrotta, R.V.V. Petrescu, C. Misiano and F.I.T. Petrescu et al., 2016h. From structural colors to super-hydrophobicity and achromatic transparent protective coatings: Ion plating plasma assisted $\mathrm{TiO} 2$ and $\mathrm{SiO} 2$ nano-film deposition. Am. J. Eng. Applied Sci., 9: 1037-1045.

DOI: 10.3844/ajeassp.2016.1037.1045

Aversa, R., R.V. Petrescu, F.I.T. Petrescu and A. Apicella, 2016i. Biomimetic and evolutionary design driven innovation in sustainable products development. Am. J. Eng. Applied Sci., 9: 1027-1036.

DOI: 10.3844/ajeassp.2016.1027.1036

Aversa, R., R.V.V. Petrescu, A. Antonio and F.I.T. Petrescu, 2016j. Mitochondria are naturally micro robots-A review. Am. J. Eng. Applied Sci., 9: 991-1002. DOI: 10.3844/ajeassp.2016.991.1002

Aversa, R., R.V.V. Petrescu, A. Apicella and F.I.T. Petrescu, 2016k. We are addicted to vitamins $\mathrm{C}$ and EA review. Am. J. Eng. Applied Sci., 9: 1003-1018. DOI: $10.3844 /$ ajeassp.2016.1003.1018

Aversa, R., R.V.V. Petrescu, A. Antonio and F.I.T. Petrescu, 20161. Physiologic human fluids and swelling behavior of hydrophilic biocompatible hybrid ceramo-polymeric materials. Am. J. Eng. Applied Sci., 9: 962-972. DOI: 10.3844/ajeassp.2016.962.972

Busch, R., 2000. The thermophysical properties of bulk metallic glass-forming liquids. JOM, 52: 39-42. DOI: $10.1007 / \mathrm{s} 11837-000-0160-7$

Cai, A.H., X. Xiong, Y. Liu, W.K. An and G.J. Zhou et al., 2012. Corrosion behavior of $\mathrm{Cu}_{55} \mathrm{Zr}_{35} \mathrm{Ti}_{10}$ metallic glass in the chloride media. Mater. Chem. Phys., 134: 938-944.

DOI: 10.1016/j.matchemphys.2012.03.094

Cubaud, T. and T.G. Mason, 2012, Interacting viscous instabilities in microfluidic systems. Soft Matter, 8: 10573-10582. DOI: 10.1039/C2SM25902H

Debenedetti, P.G. and F.H. Stillinger, 2001. Supercooled liquids and the glass transition. Nature, 410: 259-267. DOI: $10.1038 / 35065704$ 
Eckert, J., N. Mattern, M. Zinkevitch and M. Seidel, 1998. Crystallization behavior and phase formation in $\mathrm{Zr}-\mathrm{Al}-\mathrm{Cu}-\mathrm{Ni}$ metallic glass containing oxygen. Mater. Trans. JIM, 39: 623-632. DOI: 10.2320/matertrans 1989.39.623

Eyring, H, 1936. Viscosity, plasticity and diffusion as examples of absolute reaction rates. J. Chem. Phys., 4: 283-291. DOI: 10.1063/1.1749836

Glasstone, S., K.J. Laidler and H. Eyring, 1941. The Theory of Rate Pro-Cesses. The Kinetics of Chemical Reactions, Viscosity, Diffusion and Electrochemical Phenomena. McGraw-Hill Book Company, inc., New York, pp: 611.

Gostin, P.F., A. Gebert and L. Schultz, 2010. Comparison of the corrosion of bulk amorphous steel with conventional steel. Corros. Sci., 52: 273-281. DOI: 10.1016/j.corsci.2009.09.016

Huang, Y., P. Xue, S. Guo, Y. Wu and X. Cheng et al., 2016. Liquid-solid joining of bulk metallic glasses. Sci. Rep., 6: 30674-30674. PMID: 27471073

Iida, T. and R.I.L. Guthrie, 1988. The Physical Properties of Liquid Metals. 1st Edn., Clarendon Press, Oxford, ISBN-10: 0198563310, pp: 288.

Inoue, A. and A. Takeuchi, 2011. Recent development and application products of bulk glassy alloys. Acta Mater., 59: 2243-2267.

DOI: $10.1016 /$ j.actamat.2010.11.027

Jiang, Q.K., X.D. Wang, X.P. Nie, G.Q. Zhang and $\mathrm{H}$. Ma et al., 2008. $\mathrm{Zr}-(\mathrm{Cu}, \mathrm{Ag})-\mathrm{Al}$ bulk metallic glasses. Acta Mater., 56: 1785-1796. DOI: 10.1016/j.actamat.2007.12.030

Kanugo, B.P., S.C. Gladeb, P. Asoka-Kumarb and K.M. Floresa, 2004. Characterization of free volume changes associated with shear band formation in $\mathrm{Zr}$ and $\mathrm{Cu}$-based bulk metallic glasses. Intermetallics, 12: 1073-1080. DOI: 10.1016/j.intermet.2004.04.033

Kumar, G., H.X. Tang and J. Schroers, 2009. Nanomoulding with amorphous metals. Nature, 457: 868-872. DOI: $10.1038 /$ nature 07718

Lee, S.W., M.Y. Huh, E. Feury and J.C. Lee, 2006. Crystallization-induced plasticity of $\mathrm{Cu}-\mathrm{Zr}$ containing bulk amorphous alloys. Acta Mater., 54: 349-355. DOI: 10.1016/j.actamat.2005.09.007

Liu, C.T., M.F. Chisholm and M.K. Miller, 2002. Oxygen impurity and microalloying effect in a $\mathrm{Zr}$ based bulk metallic glass alloy. Intermetallics, 10: 1105-1112. DOI: 10.1016/S0966-9795(02)00131-0

Liu, Y.H., G. Wang, R.J. Wang, D.Q. Zhao and M.X. Pan et al., 2007. Super plastic bulk metallic glasses at room temperature. Science, 315: 1385-1388. DOI: $10.1126 /$ science. 1136726

Mattern, N., U. Kuhn, H. Hermann, H. Ehrenberg and J. Neuefeind, 2002. Short-range order of $\mathrm{Zr}_{62-\mathrm{x}} \mathrm{Ti}_{\mathrm{x}} \mathrm{Al}_{10} \mathrm{Cu}_{20} \mathrm{Ni}_{8}$ bulk metallic glasses. Acta Mater., 50: 305-314.

DOI: $10.1016 / \mathrm{S} 1359-6454(01) 00341-\mathrm{X}$
Morito, N. and T. Egami, 1984. Internal friction and reversible structural relaxation in the metallic glass $\mathrm{Fe}_{32} \mathrm{Ni}_{36} \mathrm{Crl}_{4} \mathrm{P}_{12} \mathrm{~B}_{6}$. Acta Metall., 32: 603-613.

DOI: $10.1016 / 0001-6160(84) 90071-3$

Schroers, J., 2010a. Processing of bulk metallic glass. Adv. Mater., 22: 1566-1597. DOI: $10.1002 /$ adma.200902776

Schroers, J., 2010b. Processing of bulk metallic glass. Adv. Mater., 21: 1566-1597. DOI: 10.1002/adma.200902776

Schroers, J., A. Masuhr, W.L. Johnson and R. Busch, 1999. Pronounced asymmetry in the crystallization behavior during constant heating and cooling of a bulk metallic glass-forming liquid. Phys. Rev. BCondensed Matter Mater. Phys., 60: 11855-11858. DOI: 10.1103/PhysRevB.60.11855

Shen, Y.T., L.Q. Xing and K.F. Kelton, 2005. Formation and crystallization of $\mathrm{ZrCuTi}$ metallic glasses. Philos. Mag., 85: 3673-3682.

DOI: $10.1080 / 14786430500156724$

Sorrentino, R., R. Aversa, V. Ferro, T. Auriemma and F. Zarone et al., 2007. Three-dimensional finite element analysis of strain and stress distributions in endodontically treated maxillary central incisors restored with diferent post, core and crown materials. Dental Mater., 23: 983-993.

DOI: 10.1016/j.dental.2006.08.006

Tam, M.K., S.J. Pang and C.H. Shek, 2007. Corrosion behavior and glass-forming ability of $\mathrm{Cu}-\mathrm{Zr}-\mathrm{Al}-\mathrm{Nb}$ alloys. J. Non-Cryst. Solids, 353: 3596-3599.

DOI: 10.1016/j.jnoncrysol.2007.05.119

Trachenko, K., 2008. The vogel-fulcher-tammann law in the elastic theory of glass transition. J. NonCrystalline Solids, 354: 3903-3906.

DOI: $10.1016 /$ j.jnoncrysol.2008.05.021

Wang, W.H., 2007. Roles of minor additions in formation and properties of bulk metallic glasses. Prog. Mater. Sci., 52: 540-596. DOI: $10.1016 /$ j.pmatsci.2006.07.003

Wang, W.H., D. Dong and C.H. Shek, 2004. Bulk metallic glasses. Mater. Sci. Eng. R, 44: 45-89. DOI: 10.1016/j.mser.2004.03.001

Way, C., P. Wadhwa and R. Busch, 2007. The influence of shear rate and temperature on the viscosity and fragility of the $\mathrm{Zr}_{41.2} \mathrm{Til}_{3.8} \mathrm{Cu}_{12.5} \mathrm{Ni}_{10.0} \mathrm{Be}_{22.5}$ metallicglass-forming liquid. Acta Mater., 55: 2977-2983. DOI: 10.1016/j.actamat.2006.12.032 